\title{
Active Sliding Mode for Synchronization of a Wide Class of Four-Dimensional Fractional-Order Chaotic Systems
}

\author{
Bin Wang, ${ }^{1}$ Yuangui Zhou, ${ }^{2}$ Jianyi Xue, ${ }^{1}$ and Delan $\mathrm{Zhu}^{1}$ \\ ${ }^{1}$ Department of Electrical Engineering, College of Water Resources and Architectural Engineering, Northwest A\&F University, \\ Yangling, Shaanxi 712100, China \\ ${ }^{2}$ School of Power and Mechanical Engineering, Wuhan, University, Wuhan, Hubei 430072, China
}

Correspondence should be addressed to Delan Zhu; dlzhu@126.com

Received 8 January 2014; Accepted 27 February 2014; Published 19 March 2014

Academic Editors: A. El-Sayed and C. Join

Copyright (C) 2014 Bin Wang et al. This is an open access article distributed under the Creative Commons Attribution License, which permits unrestricted use, distribution, and reproduction in any medium, provided the original work is properly cited.

\begin{abstract}
We focus on the synchronization of a wide class of four-dimensional (4-D) chaotic systems. Firstly, based on the stability theory in fractional-order calculus and sliding mode control, a new method is derived to make the synchronization of a wide class of fractional-order chaotic systems. Furthermore, the method guarantees the synchronization between an integer-order system and a fraction-order system and the synchronization between two fractional-order chaotic systems with different orders. Finally, three examples are presented to illustrate the effectiveness of the proposed scheme and simulation results are given to demonstrate the effectiveness of the proposed method.
\end{abstract}

\section{Introduction}

Chaos synchronization is the concept of closeness of the frequencies between different periodic oscillations generated by two chaotic systems, one of which is the master and the other is the slave. Since the pioneering work of Pecora and Carroll [1] who proposed a method to synchronize two identical chaotic systems, chaos synchronization has attracted a lot of attention in a variety of research fields over the last two decades. This is because chaos synchronization can be used in many areas such as physics, engineering, and particularly in secure communication [2-5].

Many methods have been proposed to synchronize chaotic systems including active control [6], back-stepping control [7], linear feedback control [8], adaptive control theory [9], sliding mode control [10, 11], and fuzzy control [12]. For example, Bhalekar and Daftardar-Gejji [13] used active control for the problem of synchronization of fractional-order Liu system with fractional-order Lorenz system. Based on the idea of tracking control and stability theory of fractional-order systems, Zhou and Ding [14] designed a controller to synchronize the fractional-order Lorenz chaotic system via fractional-order derivative. Zhang and Yang [15] dealt with the lag synchronization of fractionalorder chaotic systems with uncertain parameters. Projective synchronization of a class of fractional-order hyperchaotic system with uncertain parameters was studied by Bai et al. [16] as well, but the derivative orders of the state in response system was the same with drive system. Chen et al. [17] designed a sliding mode controller for a class of fractionalorder chaotic systems.

However, most of the above-mentioned work on chaos synchronization has focused on fractional-order chaos and integer-order systems, respectively. To the best of our knowledge, there has been little information available about the synchronization between chaotic fractional-order system and integer-order system or chaotic systems with noncommensurate chaotic orders.

Motivated by the above discussion, the synchronization of a class of 4-D chaotic systems with nonidentical chaotic orders has been reported. There are three advantages which make our approach attractive. First, based on the thought of sliding mode control and stability theorems in the fractional calculus, a new active sliding mode is presented. Second, the method is designed for a wide class of systems, which means its universal applicability. Third, the method guarantees that 
the synchronization between an integer-order system and a fractional-order system and the synchronization between two fractional-order chaotic systems with different orders. As a special case, it is also capable of the synchronization between two integer-order chaotic systems. In reality, the controller is expected to be easier to realize the achievement of synchronization.

The rest of the paper is organized as follows: in Section 2, basic definitions of fractional-order calculus and its approximation method are presented. Section 3 is about the brief description of a wide class of 4-D fractional-order chaotic systems. In Section 4, a new active sliding mode controller is presented which combines the active control and sliding mode control. The stability analysis for fractional-order systems is presented also. In Section 5, three typical examples are given and numerical simulations results are shown. The brief comments and conclusions are drawn in Section 6.

\section{Fractional-Order Calculus}

2.1. Definitions. For the fractional calculus operator, there are two commonly used definitions: Riemann-Liouville definition and Caputo definition, which are listed here for the sake of clarity.

Definition 1. The Riemann-Liouville fractional integral can be described as

$$
J_{t 0}^{q} f(x)=\frac{1}{\Gamma(q)} \int_{t 0}^{t}(t-\tau)^{q-1} f(\tau) d \tau
$$

where $q \in R^{+}, f: R \rightarrow R, J_{t 0}^{q}$ denotes $q$-order integral operator, $\Gamma(\cdot)$ means the Gamma function, and $\Gamma(q)=$ $\int_{0}^{\infty} t^{q-1} e^{-t} d t$

Definition 2. The Riemann-Liouville fractional derivative is given by

$$
\begin{aligned}
& D_{t 0}^{q} f(t) \\
& \quad=D_{t 0}^{n} J_{t 0}^{n-q} f(t) \\
& \quad= \begin{cases}\frac{d^{n}}{d t^{n}}\left[\frac{1}{\Gamma(n-q)} \int_{t 0}^{t}(t-\tau) f(\tau) d \tau\right], & n-1<q<n, \\
\frac{d^{n}}{d t^{n}} f(t), & q=n,\end{cases}
\end{aligned}
$$

where $D_{t 0}^{q}$ denotes $q$-order Riemann-Liouville derivative operator.
Definition 3. The Caputo derivative can be written by

$$
\begin{aligned}
{ }_{0}^{c} D_{t 0}^{q} f(t) \\
\quad=J_{t 0}^{n-q} D_{t 0}^{n} f(t) \\
\quad= \begin{cases}\frac{1}{\Gamma(n-q)} \int_{t 0}^{t}(t-\tau)^{n-q-1} f^{(n)}(\tau) d \tau, & n-1<q<n \\
\frac{d^{n}}{d t^{n}} f(t), & q=n,\end{cases}
\end{aligned}
$$

where ${ }_{0}^{c} D_{t 0}^{q}$ denotes $q$-order Caputor derivative operator, which can be simplified as $D^{q}$.

From the point of view of mathematics, the RiemannLiouville fractional derivative is widely used in theoretical study. However, the Caputo derivative can be easily given initial conditions, so it has a popular application in engineering. In this paper, we will use the Caputo derivative.

2.2. Approximation Method. To get the solutions of the nonlinear fractional-order differential equations, we employ an improved version of Adams-Bashforth-Moulton algorithm, which is a very useful time domain approximation method for fractional-order differential equations. Here, consider the following differential equation with initial condition:

$$
\begin{gathered}
D^{q} x(t)=f(t, x(t)), \quad 0 \leq t<T, \\
x^{(k)}(0)=x_{0}^{k}, \quad k=0,1,2, \ldots, n-1 .
\end{gathered}
$$

This differential equation is equivalent to Volterra integral equation:

$$
x(t)=\sum_{k=0}^{[q]-1} x_{0}^{(k)} \frac{t^{k}}{k !}+\frac{1}{\Gamma(q)} \int_{0}^{t}(t-\tau)^{q-1} f(\tau, x(\tau)) d \tau .
$$

Now, set

$$
h=\frac{T}{N}, \quad t_{j}=j h, \quad j=0,1, \ldots, N \in Z^{+} .
$$

Then (5) can be discretized as follows:

$$
\begin{aligned}
x_{h}\left(t_{n+1}\right)= & \sum_{k=0}^{[q]-1} x_{0}^{(k)} \frac{t_{n+1}^{k}}{k !}+\frac{h^{q}}{\Gamma(q+2)} f\left(t_{n+1}, x_{h}^{p}\left(t_{n+1}\right)\right) \\
& +\frac{h^{q}}{\Gamma(q+2)} \sum_{j=0}^{n} a_{j, n+1} f\left(t_{j}, x_{h}\left(t_{j}\right)\right),
\end{aligned}
$$

where predicted value $x_{h}\left(t_{n+1}\right)$ is determined by

$$
x_{h}^{p}\left(t_{n+1}\right)=\sum_{k=0}^{[q]-1} x_{0}^{(k)} \frac{t_{n+1}^{k}}{k !}+\frac{1}{\Gamma(q)} \sum_{j=0}^{n} b_{j, n+1} f\left(t_{j}, x_{h}\left(t_{j}\right)\right)
$$


in which

$$
\begin{aligned}
& a_{j, n+1}= \begin{cases}n^{q+1}-(n-q)(n+1)^{q}, & j=0 \\
(n-j+2)^{q+1}+(n-j)^{q+1} & \\
-2(n-j+1)^{q+1}, & 1 \leq j \leq n \\
1, & j=n+1,\end{cases} \\
& b_{j, n+1}=\frac{h^{q}}{q}\left((n-j+1)^{q}-(n-j)^{q}\right), \quad 0 \leq j \leq n .
\end{aligned}
$$

The estimation error of this approximation is given as follows:

$$
\max _{j=0,1, \ldots, N}\left|x\left(t_{j}\right)-x_{h}\left(t_{j}\right)\right|=O\left(h^{p}\right)
$$

where $p=\min (2,1+q)$. So by applying the aforementioned method, numerical solution of a fractional-order system can be obtained. Give the following 4-D fractional system:

$$
\begin{aligned}
& D^{q_{1}} x=f_{1}(x, y, z, w), \\
& D^{q_{2}} y=f_{2}(x, y, z, w), \\
& D^{q_{3}} z=f_{3}(x, y, z, w), \\
& D^{q_{4}} w=f_{4}(x, y, z, w),
\end{aligned}
$$

with $0<q_{i} \leq 1(i=1,2,3,4)$ and initial conditions $\left(x_{0}, y_{0}, z_{0}, w_{0}\right)$. Applying the above method, the system can be discretized as follows:

$$
\begin{aligned}
x_{n+1}= & x_{0}+\frac{h^{q 1}}{\Gamma\left(q_{1}+2\right)} \\
\times & {\left[f_{1}\left(x_{n+1}^{p}, y_{n+1}^{p}, z_{n+1}^{p}, w_{n+1}^{p}\right)\right.} \\
& \left.+\sum_{j=0}^{n} \alpha_{1, j, n+1} f_{1}\left(x_{j}, y_{j}, z_{j}, w_{j}\right)\right], \\
y_{n+1}= & y_{0}+\frac{h^{q 2}}{\Gamma\left(q_{2}+2\right)} \\
\times & {\left[f_{2}\left(x_{n+1}^{p}, y_{n+1}^{p}, z_{n+1}^{p}, w_{n+1}^{p}\right)\right.} \\
& \left.+\sum_{j=0}^{n} \alpha_{2, j, n+1} f_{2}\left(x_{j}, y_{j}, z_{j}, w_{j}\right)\right],
\end{aligned}
$$

$$
\begin{aligned}
z_{n+1}= & z_{0}+\frac{h^{q 3}}{\Gamma\left(q_{3}+2\right)} \\
\times & {\left[f_{3}\left(x_{n+1}^{p}, y_{n+1}^{p}, z_{n+1}^{p}, w_{n+1}^{p}\right)\right.} \\
& \left.+\sum_{j=0}^{n} \alpha_{3, j, n+1} f_{3}\left(x_{j}, y_{j}, z_{j}, w_{j}\right)\right], \\
w_{n+1}=w_{0}+\frac{h^{q 4}}{\Gamma\left(q_{4}+2\right)} & {\left[\begin{array}{l}
f_{4}\left(x_{n+1}^{p}, y_{n+1}^{p}, z_{n+1}^{p}, w_{n+1}^{p}\right) \\
\left.+\sum_{j=0}^{n} \alpha_{4, j, n+1} f_{4}\left(x_{j}, y_{j}, z_{j}, w_{j}\right)\right],
\end{array}\right.}
\end{aligned}
$$

where

$$
\begin{aligned}
& x_{n+1}^{p}=x_{0}+\frac{1}{\Gamma\left(q_{1}\right)} \sum_{j=0}^{n} \beta_{1, j, n+1} f_{1}\left(x_{j}, y_{j}, z_{j}, w_{j}\right), \\
& y_{n+1}^{p}=y_{0}+\frac{1}{\Gamma\left(q_{2}\right)} \sum_{j=0}^{n} \beta_{2, j, n+1} f_{2}\left(x_{j}, y_{j}, z_{j}, w_{j}\right) \text {, } \\
& z_{n+1}^{p}=z_{0}+\frac{1}{\Gamma\left(q_{3}\right)} \sum_{j=0}^{n} \beta_{3, j, n+1} f_{3}\left(x_{j}, y_{j}, z_{j}, w_{j}\right), \\
& w_{n+1}^{p}=w_{0}+\frac{1}{\Gamma\left(q_{4}\right)} \sum_{j=0}^{n} \beta_{4, j, n+1} f_{4}\left(x_{j}, y_{j}, z_{j}, w_{j}\right), \\
& \alpha_{i, j, n+1}= \begin{cases}n^{q_{i}+1}-\left(n-q_{i}\right)(n+1)^{q i}, & j=0, \\
(n-j+2)^{q_{i}+1}+(n-j)^{q_{i}+1} & \\
-2(n-j+1)^{q_{i}+1}, & 1 \leq j \leq n, \\
1, & j=n+1,\end{cases} \\
& \beta_{i, j, n+1}=\frac{h^{q_{i}}}{q_{i}}\left((n-j+1)^{q_{i}}-(n-j)^{q_{i}}\right), \quad 0 \leq j \leq n .
\end{aligned}
$$

\section{System Description}

We consider a wide class of 4 -D chaotic systems. And thus a master system and a slave system are described as

$$
\begin{gathered}
D^{q_{m}} x=A x+f(x), \\
D^{q_{s}} y=B y+g(y)+U(x, y),
\end{gathered}
$$

where $x(t), y(t) \in R^{4}$ are the four-dimensional state vectors for the master and slave systems, respectively. $A, B \in R^{4 \times 4}$ are the linear parts for the systems. $f, g: R_{4} \rightarrow R_{4}$ are 
nonlinear parts for the systems. $q_{m i}(i=1,2,3,4)$ and $q_{s i}(i=$ $1,2,3,4)$ are fractional orders for each state of master and slave systems satisfying $0<q_{m i} \leq 1,0<q_{s i} \leq 1$, and for $q_{m i}=1(i=1,2,3,4), q_{s i}=1(i=1,2,3,4)$, system $(14)$ and system (15) are named as integer-order systems. $U(x, y)$ is the controller, added on the slave system, to realize the synchronization between the master and slave system. That is,

$$
\lim _{t \rightarrow \infty}\|e\|=\lim _{t \rightarrow \infty}\|y-x\|=0
$$

Our aim is to design a suitable and effective controller $U(x, y)$ such that the trajectories of the slave system asymptotically approach the master system and the synchronization between the two systems is achieved finally.

Remark 4. The fractional-order system (15) is called a commensurate fractional-order system if $q_{s 1}=q_{s 2}=q_{s 3}=q_{s 4}=$ q. Otherwise, we call the system (15) a noncommensurate fractional-order system.

\section{Active Sliding Mode Controller Design}

4.1. Active Controller Design. To obtain the control law, synchronization error is defined as

$$
e=y-\chi x
$$

where $\chi$ is the scaling factor, which is an arbitrary constant $(\chi \in R)$. We can choose the value of $\chi$ randomly to meet our demands. The synchronization is realized when $\chi=1$ and the antisynchronization is achieved when $\chi=-1$.

Based on the thought of active control, we design $U(x, y)$ to eliminate the nonlinear part of the error system. So,

$$
U(x, y)=D^{q_{s}}(\chi x)-g(y)-B(\chi x)+H u(t),
$$

where $H \in R^{4 \times 4}$ is the designed control vector. $u(t) \in R^{4 \times 1}$ is the new control input, which will be designed later. Substitute (18) into (15), and then one gets

$$
D^{q_{s}} e=B e+H u(t) .
$$

4.2. Sliding Mode Controller Design. To design a sliding mode controller, there are two steps: first, a sliding surface should be constructed that represents a desired system dynamics, and, next, a switching control law should be developed such that a sliding mode exists on every point of the sliding surface, and any states outside the surface are driven to reach the surface in a finite time. As a choice for the switching surface, we have

$$
S(t)=K D^{q_{s}-1} e(t)+\int(A-L) e(\tau) d \tau,
$$

where $K$ and $L$ are the designed parameter matrixes.

The sliding mode controller is designed as

$$
u(t)=u_{\mathrm{eq}}+u_{d}
$$

where $u_{\text {eq }}$ is the equivalent control law and $u_{d}$ is the switching control law.
In the sliding mode, the sliding surface and its derivative must satisfy

$$
S(t)=\dot{S}(t)=0 .
$$

Considering (19), (20), and (22), one gets

$$
\begin{aligned}
\dot{S} & =K D^{q_{s}} e(t)+(A-L) e(t) \\
& =K D^{q_{s}} e(t)+(A-L) e(t) \\
& =K[B e+H u(t)]+(A-L) e=0 .
\end{aligned}
$$

Now, the equivalent control law can be got as

$$
u_{\mathrm{eq}}=-H^{-1}\left[K^{-1}(A-L)+B\right] e .
$$

To satisfy the sliding mode condition, the discontinuous reaching law is chosen as follows:

$$
\dot{S}=-\xi \cdot \operatorname{sat}\left(\frac{S}{\delta}\right)
$$

where $\xi$ and $\delta$ are the positive constants and

$$
\text { sat }(x)= \begin{cases}\operatorname{sign}(x), & |x|>1 \\ x, & |x| \leq 1,\end{cases}
$$

where $\operatorname{sign}(x)$ is the sign function of $x$, if $x>0, \operatorname{sign}(x)=1$; if $x=0, \operatorname{sign}(x)=0$; if $x<0, \operatorname{sign}(x)=-1$.

Consider (23) and (25), one gets the control input

$$
u(t)=-H^{-1}\left[K^{-1}(A-L)+B\right] e-(K H)^{-1} \xi \operatorname{sat}\left(\frac{S}{\delta}\right) .
$$

Let $u_{d}=-(K H)^{-1} \xi \operatorname{sat}(S / \delta),(27)$ just be the $u(t)=u_{\text {eq }}+u_{d}$.

Substitute (27) into (18), the total control law can be got as

$$
\begin{aligned}
U(x, y)= & D^{q_{s}}(\chi x)-g(y)-B y \\
& -K^{-1}(A-L) e-K^{-1} \xi \operatorname{sat}\left(\frac{S}{\delta}\right)
\end{aligned}
$$

and the error system (19) is

$$
D^{q_{s}} e=-K^{-1}\left[(A-L) e+\xi \text { sat }\left(\frac{S}{\delta}\right)\right] .
$$

\subsection{Stability Analysis}

Theorem 5 (see [18]). Consider the following linear fractional order system:

$$
D^{q} x=A x, \quad x(0)=x_{0},
$$

where $q \in(0,1], A \in R^{n \times n}$, and $x=\left(x_{1}, x_{2}, \ldots, x_{n}\right)^{T}$. System (30) is asymptotically stable if and only if $\left|\arg \left(\lambda_{i}\right)\right|>q \pi / 2$ is satisfied for all eigenvalues $\lambda_{i}$ of matrix $A$. Besides, this system is stable if and only if $\left|\arg \left(\lambda_{i}\right)\right| \geq q \pi / 2$ is satisfied for all eigenvalues $\lambda_{i}$ of matrix $A$ and those critical eigenvalues that satisfy the condition $\left|\arg \left(\lambda_{i}\right)\right|=q \pi / 2$ have geometric multiplicity one. 


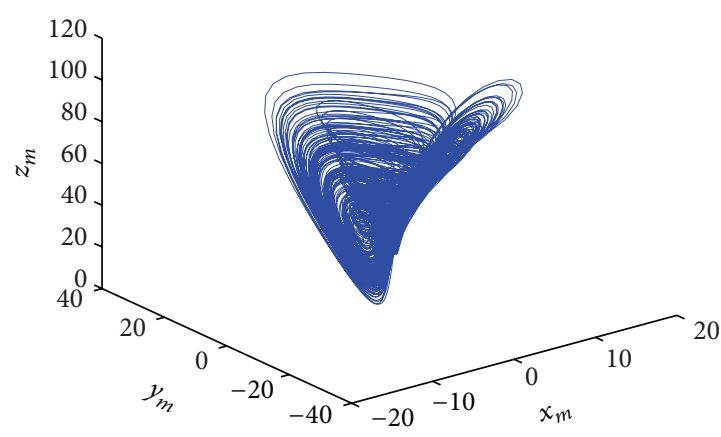

(a) $x_{m}-y_{m}-z_{m}$

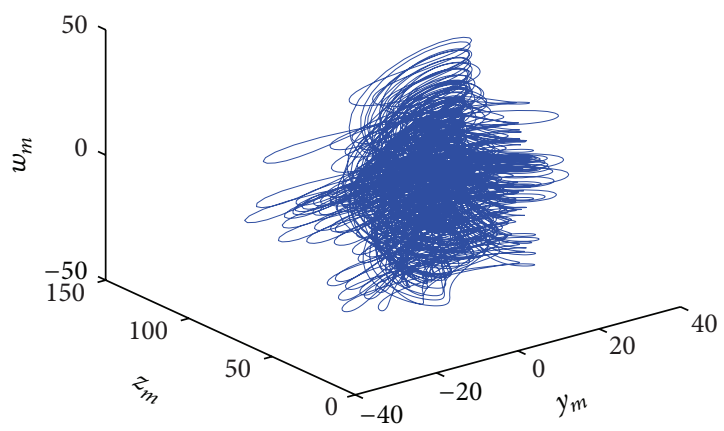

(b) $y_{m}-z_{m}-w_{m}$

Figure 1: Phase portraits of the integer-order Liu system.

Theorem 6 (see [18]). Consider a system given in the following linear state space form with inner dimension $n$ :

$$
D^{q} x=A x+B u, \quad y=C x,
$$

where $q \in(0,1], x \in R^{n}, y \in R^{p}$, and $A \in R^{n \times n}$. Assuming that the triplet $(A, B, C)$ is minimal, system (31) is boundedinput bounded-output stable when $|\arg (\operatorname{eig}(A))|>q \pi / 2$.

According to Theorems 5 and 6, in the sliding phase, error system (29) is a linear fractional-order system with bounded input $-K^{-1} \xi \operatorname{sat}(S / \delta)$; if $\left|\arg \left(\operatorname{eig}\left(-K^{-1}(A-L)\right)\right)\right|>q_{s} \pi / 2$, the error system (29) is asymptotically stable. It can be shown that choosing appropriate values of $K$ and $L$ can make the error dynamics stable.

Remark 7. Consider the control law (28); if the controller parameters $\xi>0, \delta>0$, the synchronization between system (14) and system (15) is globally asymptotically stable.

Proof. Consider a candidate Lyapunov function as

$$
V=\frac{1}{2} S^{2}
$$

So,

$$
\dot{V}=S \dot{S}=S\left(-\xi \cdot \operatorname{sat}\left(\frac{S}{\delta}\right)\right)=-\xi \cdot S \cdot \operatorname{sat}\left(\frac{S}{\delta}\right) .
$$

Then, outside the boundary layer, that is, $|S|>\delta$, we have

$$
\dot{V}=-\xi \cdot S \cdot \operatorname{sign}\left(\frac{S}{\delta}\right) \leq-\xi\|S\| \leq 0
$$

Otherwise, inside the boundary layer, that is, $|S| \leq \delta$, we have

$$
\dot{V}=-\xi \cdot S \cdot \frac{S}{\delta} \leq-\left(\frac{\xi}{\delta}\right)\|S\|^{2} \leq 0 .
$$

Since the Lyapunov function is positive definite and its derivative is negative definite, by Lyapunov stability theory, the synchronization between system (14) and system (15) is globally asymptotically stable.

\section{Numerical Simulations}

In this section, we presented three illustrative examples to verify and demonstrate the effectiveness of the proposed control scheme. The numerical simulations are carried out applying Caputo definition and a generalization AdamsBashforth-Moulton algorithm in time steps of 0.01 .

Case 1. Synchronization of the integer-order Liu system and the fractional-order Chen system.

Consider the integer-order Liu system [19] as the master system:

$$
\begin{aligned}
& D^{q_{m 1}} x_{m}=a\left(y_{m}-x_{m}\right) \\
& D^{q_{m 2}} y_{m}=b x_{m}-k x_{m} z_{m}+w_{m} \\
& D^{q_{m 3}} z_{m}=-c z_{m}+h x_{m}^{2}, \\
& D^{q_{m 4}} w_{m}=-d x_{m}
\end{aligned}
$$

where $(a, b, c, d, h, k)=(10,40,2.5,10.6,4,1)$ and $q_{m i}=1$ $(i=1,2,3,4)$. The chaotic behavior for the master system (36) is shown in Figure 1 with the initial conditions: $\left[x_{m 0}, y_{m 0}, z_{m 0}, w_{m 0}\right]^{T}=[-5,0,60,5]^{T}$.

Take the fractional-order Chen system [20] with commensurate orders as the slave system:

$$
\begin{aligned}
& D^{q_{s 1}} x_{s}=a\left(y_{s}-x_{s}\right)+w_{s}+U_{1}, \\
& D^{q_{s 2}} y_{s}=b x_{s}+c y_{s}-x_{s} z_{s}+U_{2}, \\
& D^{q_{s 3}} z_{s}=x_{s} y_{s}-d z_{s}+U_{3}, \\
& D^{q_{s 4}} w_{s}=y_{s} z_{s}+r w_{s}+U_{4},
\end{aligned}
$$

where $(a, b, c, d, r)=(35,7,12,3,0.5)$ and $q_{s i}=0.96(i=$ $1,2,3,4)$. The chaotic behavior for the slave system (37) is shown in Figure 2, with the initial conditions: $\left[x_{s 0}, y_{s 0}, z_{s 0}, w_{s 0}\right]^{T}=[7,10,20,-40]^{T}$.

For simplicity, in this paper, we make the design parameter matrix $K=\operatorname{diag}(1,1,1,1)$. Now, the parameters $\xi=5.5$ 


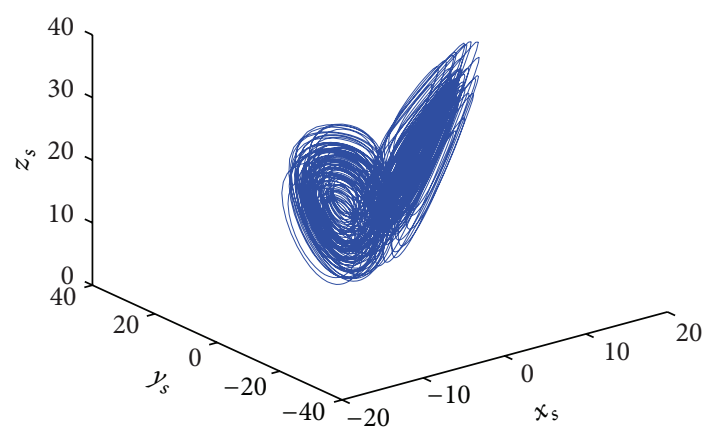

(a) $x_{s}-y_{s}-z_{s}$

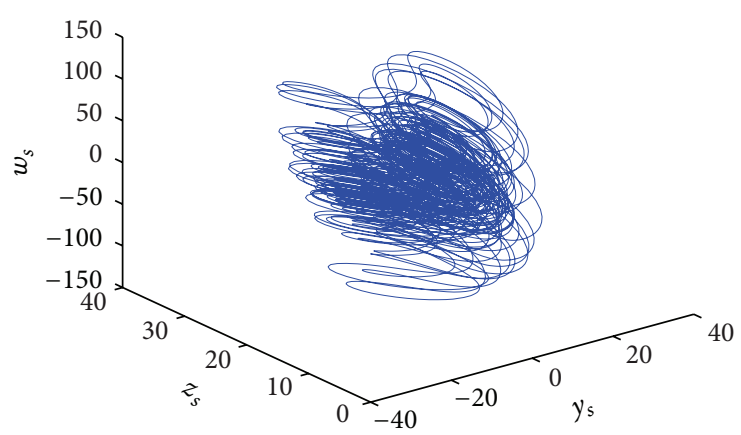

(b) $y_{s}-z_{s}-w_{s}$

Figure 2: Phase portraits of the fractional-order Chen system.

and $\delta=0.1$ are specific. The designed parameter matrix is selected as

$$
L=\left[\begin{array}{cccc}
-15 & 10 & 0 & 0 \\
40 & -7 & -5 & 0 \\
-7 & 0 & -6 & -8 \\
-10.6 & -5 & 0 & -4
\end{array}\right]
$$

and we can get the eigenvalues of the matrix - $A-$ $L),\left(\lambda_{1}, \lambda_{2}, \lambda_{3}, \lambda_{4}\right)=(-1.6252+4.7215 i,-1.6252-$ $4.7215 i,-11.2496,-5)$, which are all located in the stable region. According to the stability theory of fractional-order system, the error system can be asymptotically stable and the synchronization is achieved. is,

According to (28), we can yield the controller easily, that

$$
\begin{aligned}
U_{1}= & D^{0.96} x_{m}-\left(35\left(y_{s}-x_{s}\right)+w_{s}\right) \\
& -5 e_{1}-5.5 \mathrm{sat}\left(\frac{S_{1}}{0.1}\right), \\
U_{2}= & D^{0.96} y_{m}-\left(7 x_{s}+12 y_{s}-x_{s} z_{s}\right) \\
& -\left(7 e_{2}+5 e_{3}+e_{4}\right)-5.5 \mathrm{sat}\left(\frac{S_{2}}{0.1}\right), \\
U_{3}= & D^{0.96} z_{m}-\left(x_{s} y_{s}-3 z_{s}\right) \\
& -\left(7 e_{1}+3.5 e_{3}+8 e_{4}\right)-5.5 \mathrm{sat}\left(\frac{S_{3}}{0.1}\right), \\
U_{4}= & D^{0.96} w_{m}-\left(y_{s} z_{s}+0.5 w_{s}\right) \\
& -\left(5 e_{2}+4 e_{4}\right)-5.5 \mathrm{sat}\left(\frac{S_{4}}{0.1}\right) .
\end{aligned}
$$

The simulation results are given in Figure 3. The synchronization errors converge to zero immediately which implies the achievement of synchronization between the two systems.

Case 2. Synchronization of the noncommensurate fractional-order Lorenz system and commensurate fractional-order Lü system.
Consider the noncommensurate fractional-order Lorenz system [21] as the master system:

$$
\begin{aligned}
& D^{q_{m 1}} x_{m}=a\left(y_{m}-x_{m}\right)+w_{m}, \\
& D^{q_{m 2}} y_{m}=c x_{m}-y_{m}-x_{m} z_{m}, \\
& D^{q_{m 3}} z_{m}=x_{m} y_{m}-b z_{m}, \\
& D^{q_{m 4}} w_{m}=-y_{m} z_{m}+d w_{m},
\end{aligned}
$$

where $(a, b, c, d)=(10,8 / 3,28,-1)$ and $q_{m 1}=0.99, q_{m 2}=$ $0.98, q_{m 3}=0.97, q_{m 4}=0.98$. The chaotic behavior for the master system (40) is shown in Figure 4, with the initial conditions: $\left[x_{m 0}, y_{m 0}, z_{m 0}, w_{m 0}\right]^{T}=[0,-5,25,3]^{T}$.

Take the following commensurate fractional-order Lü system [22] as the slave system:

$$
\begin{aligned}
& D^{q_{s 1}} x_{s}=a\left(y_{s}-x_{s}\right)+w_{s} \\
& D^{q_{s 2}} y_{s}=c y_{s}-x_{s} z_{s}, \\
& D^{q_{s 3}} z_{s}=x_{s} y_{s}-b z_{s}, \\
& D^{q_{s 4}} w_{s}=x_{s} z_{s}+d w_{s}
\end{aligned}
$$

where $(a, b, c, d)=(36,3,20,-1)$ and $q_{m i}=0.95(i=1,2$, $3,4)$. The chaotic behavior for the slave system (41) is shown in Figure 5, with the initial condition: $\left[x_{s 0}, y_{s 0}, z_{s 0}, w_{s 0}\right]^{T}=$ $[-8,-10,20,13]^{T}$.

Now, the parameters $\xi=5$ and $\delta=0.1$ are specific. The designed parameter matrix is selected as

$$
L=\left[\begin{array}{cccc}
-15 & 0 & 0 & 0 \\
28 & -5 & 5 & 0 \\
0 & -2 & -4 & 0 \\
0 & 0 & -10 & -5
\end{array}\right]
$$

and we can get the eigenvalues $\left(\lambda_{1}, \lambda_{2}, \lambda_{3}, \lambda_{4}\right)=(-5,-5$, $-2.667+2.8674 i,-2.667-2.8674 i)$ of the matrix $-(A-L)$, which are all located in the stable region. 


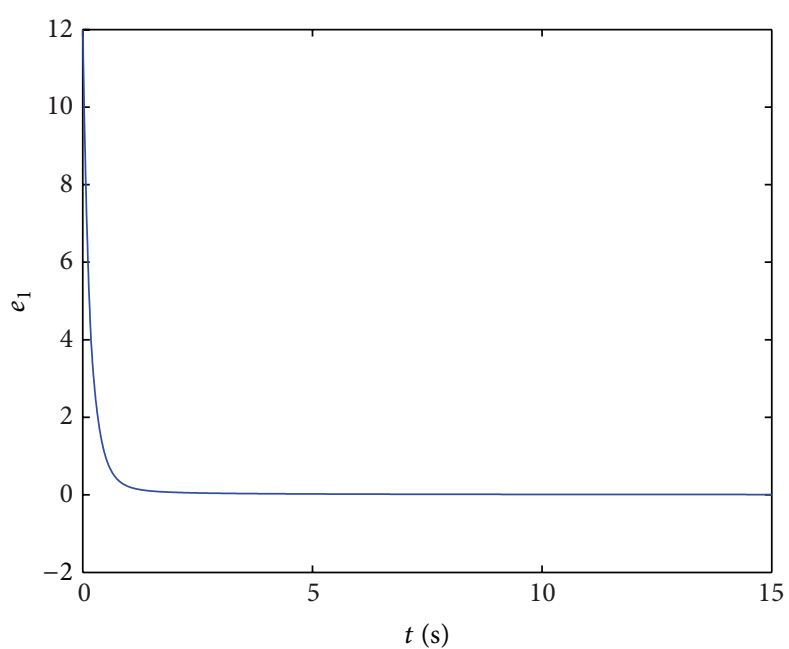

(a) $e_{1}$

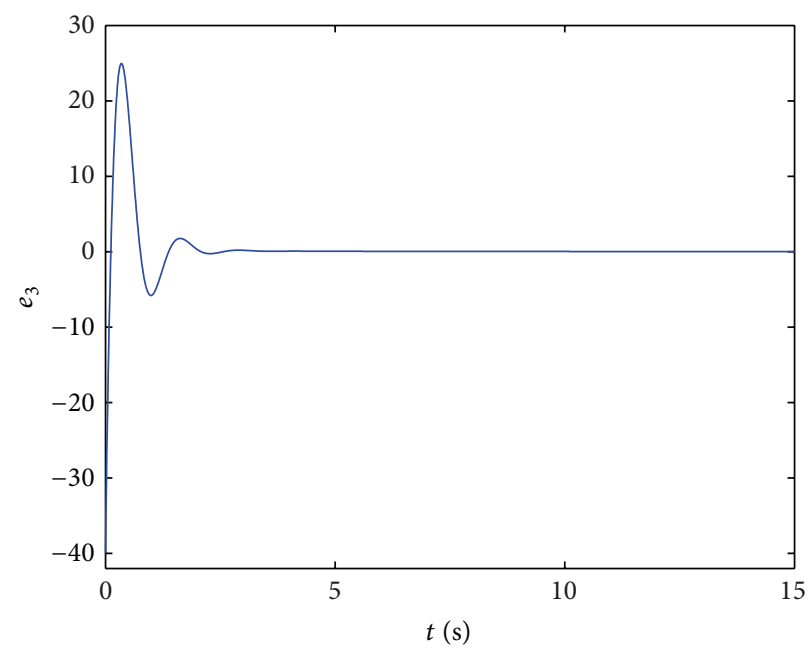

(c) $e_{3}$

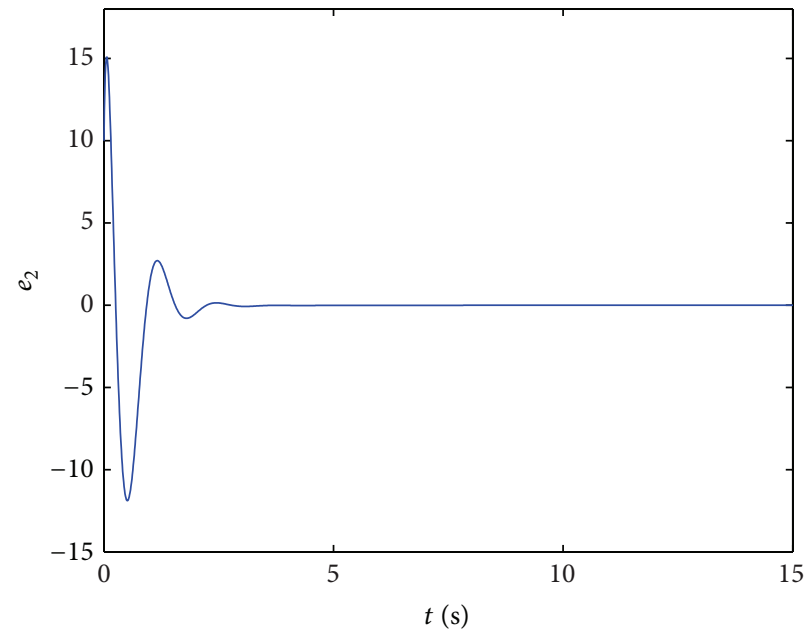

(b) $e_{2}$

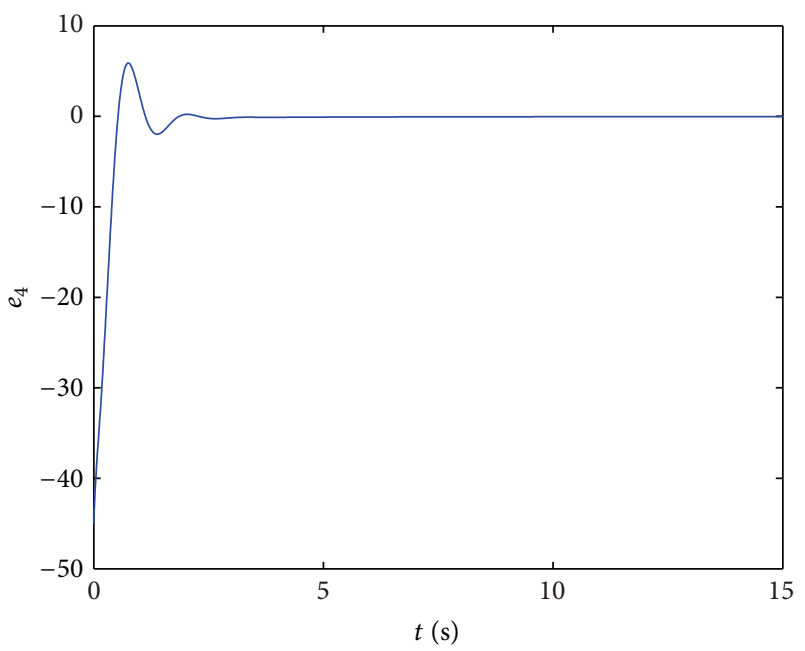

(d) $e_{4}$

FIGURE 3: Synchronization errors between the integer-order Liu system and the fractional-order Chen systems. is,

According to (28), we can yield the controller easily, that

$$
\begin{aligned}
U_{1}= & D^{0.95} x_{m}-\left(36\left(y_{s}-x_{s}\right)+w_{s}\right) \\
& -\left(5 e_{1}+10 e_{2}+e_{4}\right)-5 \text { sat }\left(\frac{S_{1}}{0.1}\right), \\
U_{2}= & D^{0.95} y_{m}-\left(20 y_{s}-x_{s} z_{s}\right) \\
& -\left(4 e_{2}-5 e_{3}\right)-5 \operatorname{sat}\left(\frac{S_{2}}{0.1}\right) \\
U_{3}= & D^{0.95} z_{m}-\left(x_{s} y_{s}-3 z_{s}\right) \\
& -\left(2 e_{2}+\frac{4}{3} e_{3}\right)-5 \operatorname{sat}\left(\frac{S_{3}}{0.1}\right), \\
U_{4}= & D^{0.95} w_{m}-\left(x_{s} z_{s}-w_{s}\right) \\
& -\left(9 e_{3}+5 e_{4}\right)-5 \text { sat }\left(\frac{S_{4}}{0.1}\right) .
\end{aligned}
$$

The simulation results are given in Figure 6. The synchronization errors converge to zero immediately which implies the achievement of synchronization between the two systems.

Case 3. Synchronization between the integer-order Liu system and the integer-order Lorenz system.

Consider the integer-order Liu system [19] as the master system, which is the same as the master system (36) in Case 1 and its chaotic attractor is also in Figure 1.

Take the following integer-order Lorenz system [23] as the slave system:

$$
\begin{aligned}
& D^{q_{s 1}} x_{r}=a\left(y_{s}-x_{s}\right)+w_{s}, \\
& D^{q_{s 2}} y_{r}=c x_{s}-y_{s}-x_{s} z_{s}, \\
& D^{q_{s 3}} z_{r}=x_{s} y_{s}-b z_{s}, \\
& D^{q_{s 4}} w_{r}=-y_{s} z_{s}+d w_{s},
\end{aligned}
$$




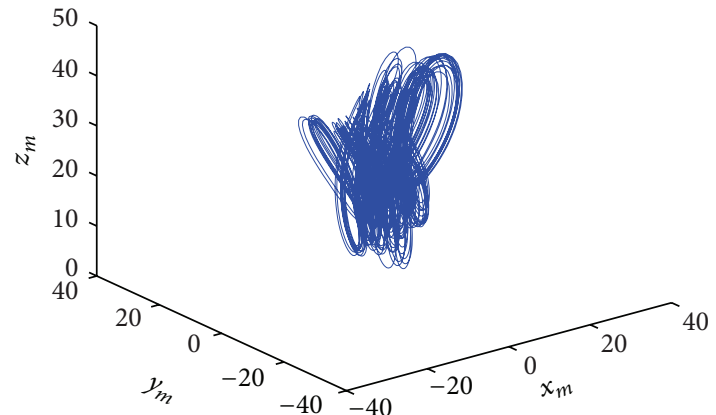

(a) $x_{m}-y_{m}-z_{m}$

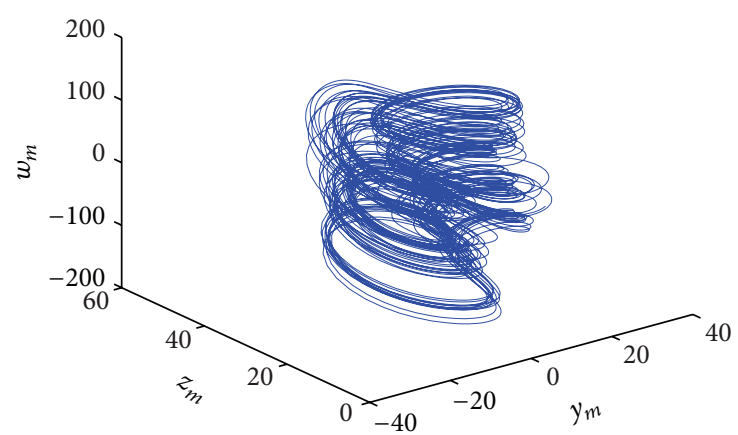

(b) $y_{m}-z_{m}-w_{m}$

FIGURE 4: Phase portraits of the fractional-order Lorenz system.

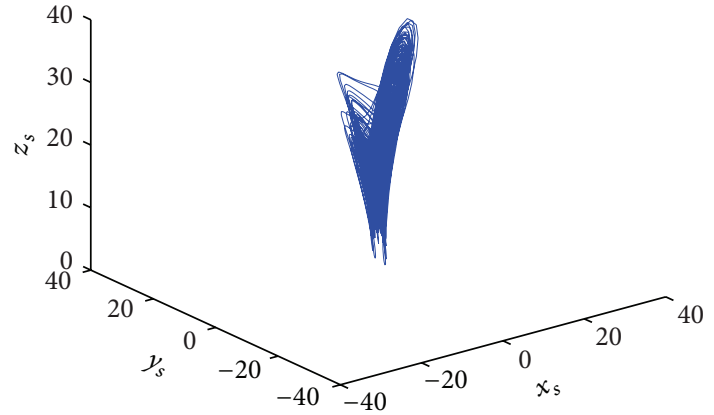

(a) $x_{s}-y_{s}-z_{s}$

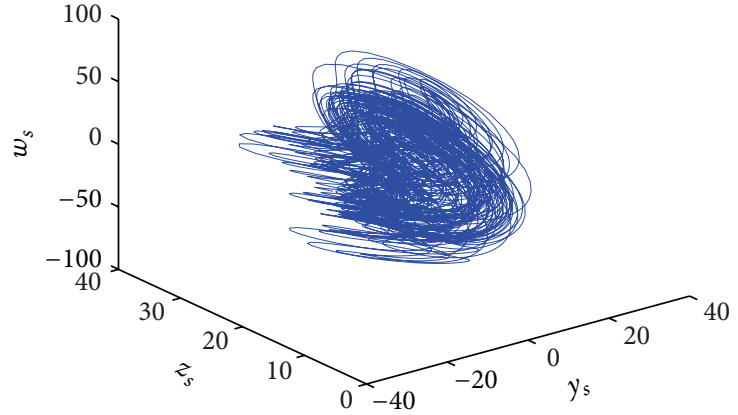

(b) $y_{s}-z_{s}-w_{s}$

Figure 5: Phase portraits of the fractional-order Lü system.

where $(a, b, c, d)=(10,8 / 3,28,-1)$ and $q_{s i}=1(i=1,2,3,4)$. The chaotic behavior for the master system (44) is shown in Figure 7, with the initial conditions: $\left[x_{s 0}, y_{s 0}, z_{s 0}, w_{s 0}\right]^{T}=$ $[-15,-6,20,-5]^{T}$.

For simplicity, make the design parameter matrix $K=$ $\operatorname{diag}(1,1,1,1)$ in this paper. Now, the parameters $\xi=4$ and $\delta=0.1$ are specific. The design parameter matrix is selected as

$$
L=\left[\begin{array}{cccc}
-20 & 10 & 0 & 0 \\
0 & -10 & 0 & 0 \\
0 & 0 & -5 & 0 \\
-15.6 & -10 & 0 & -10
\end{array}\right]
$$

and we can get the eigenvalues of the matrix $-(A-L)$ that are $\left(\lambda_{1}, \lambda_{2}, \lambda_{3}, \lambda_{4}\right)=(-6.8337,-13.1623,-10,-2.5)$, which are all located in the stable region. is,

According to (28), we can yield the controller easily, that

$$
\begin{aligned}
U_{1}= & D^{1} x_{m}-\left(10\left(y_{s}-x_{s}\right)+w_{s}\right) \\
& -10 e_{1}-4 \operatorname{sat}\left(\frac{S_{1}}{0.1}\right), \\
U_{2}= & D^{1} y_{m}-\left(28 x_{s}-y_{s}-x_{s} z_{s}\right) \\
& -\left(40 e_{1}+10 e_{2}+e_{4}\right)-4 \operatorname{sat}\left(\frac{S_{2}}{0.1}\right),
\end{aligned}
$$

$$
\begin{aligned}
U_{3}= & D^{1} z_{m}-\left(x_{s} y_{s}-\frac{8}{3} z_{s}\right) \\
& -2.5 e_{3}-4 \operatorname{sat}\left(\frac{S_{3}}{0.1}\right), \\
U_{4}= & D^{1} w_{m}-\left(-y_{s} z_{s}-w_{s}\right) \\
& -\left(5 e_{1}+10 e_{2}+10 e_{4}\right)-4 \operatorname{sat}\left(\frac{S_{4}}{0.1}\right) .
\end{aligned}
$$

The simulation results are given in Figure 8. As we can see, the synchronization errors converge to zero immediately which implies the achievement of synchronization between the two systems.

\section{Conclusion and Discussion}

In this paper, the synchronization of a wide class of 4-D chaotic systems is achieved based on a new active sliding mode control which unites the active control and sliding mode control. The proposed synchronization approach is theoretically rigorous and pervasive. Furthermore, three typical examples were shown: (1) the synchronization between the integer-order Liu system and the fractional-order Chen system, (2) the noncommensurate fractional-order Lorenz system and commensurate fractional-order Lü system, 


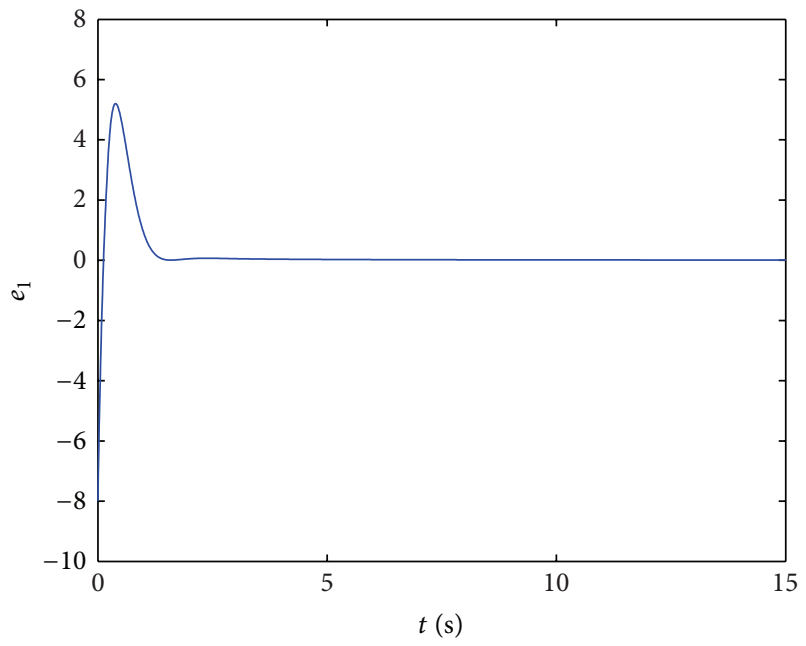

(a) $e_{1}$

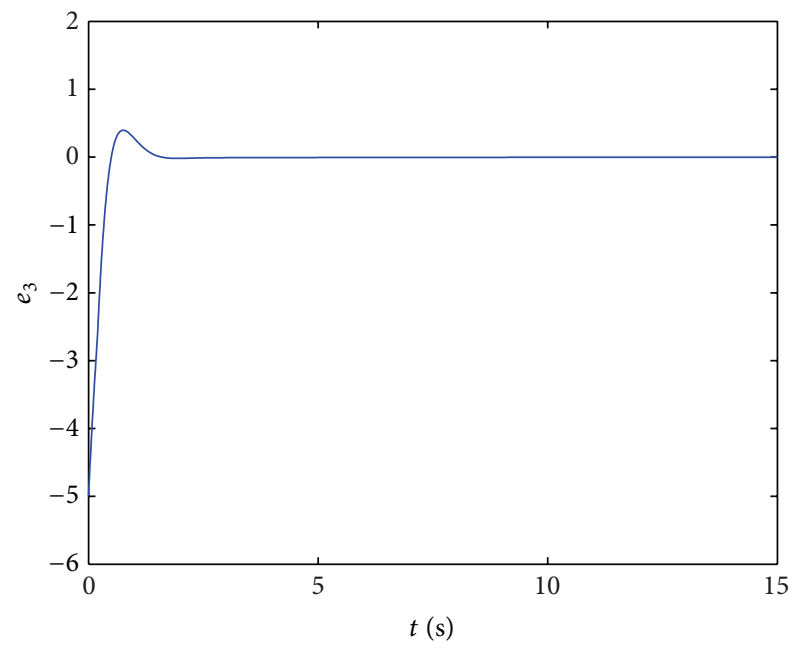

(c) $e_{3}$

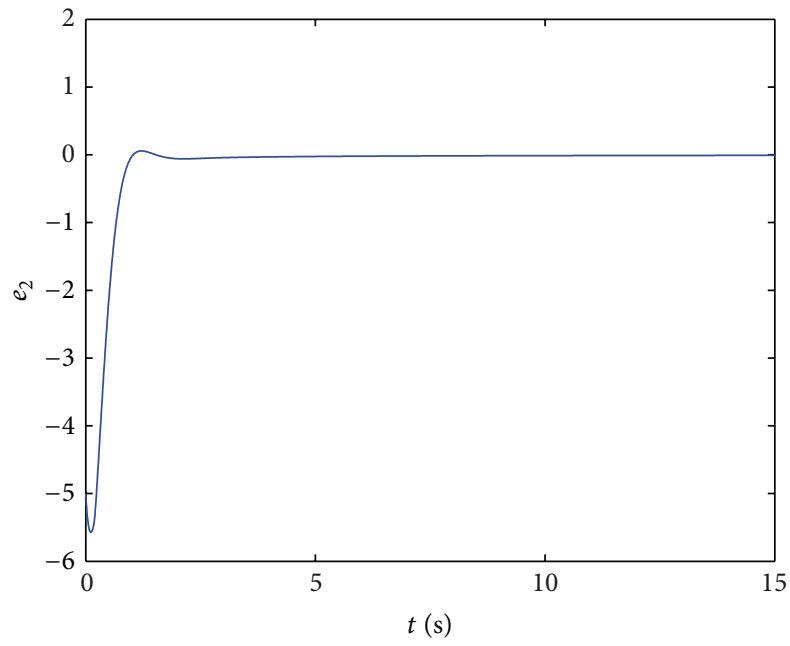

(b) $e_{2}$

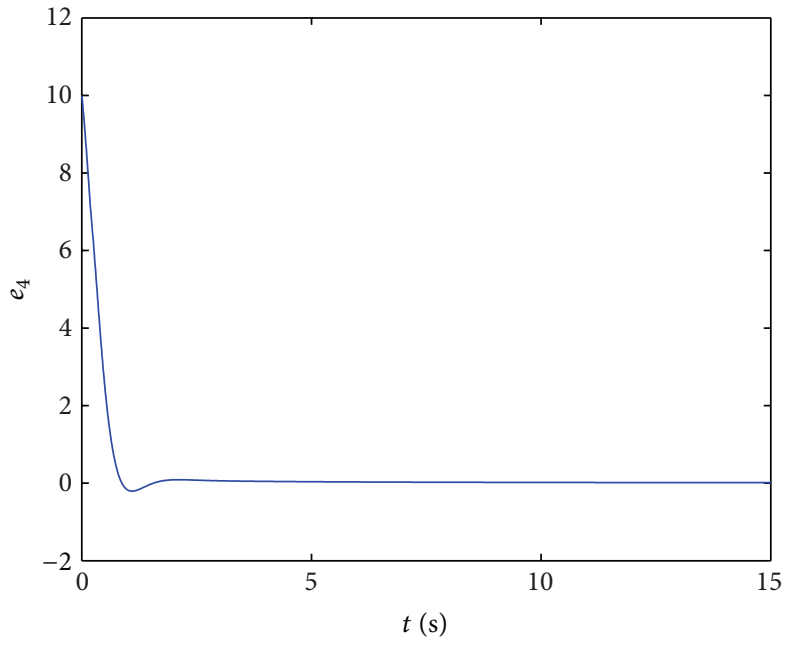

(d) $e_{4}$

FIGURE 6: Synchronization errors between the noncommensurate fractional-order Lorenz system and commensurate fractional-order Lü system.

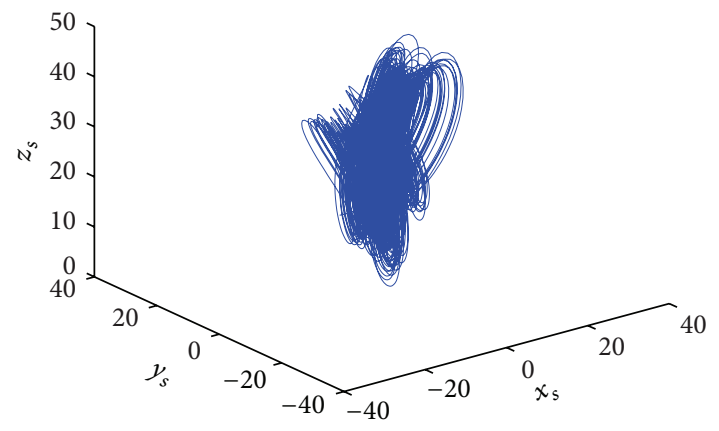

(a) $x_{s}-y_{s}-z_{s}$

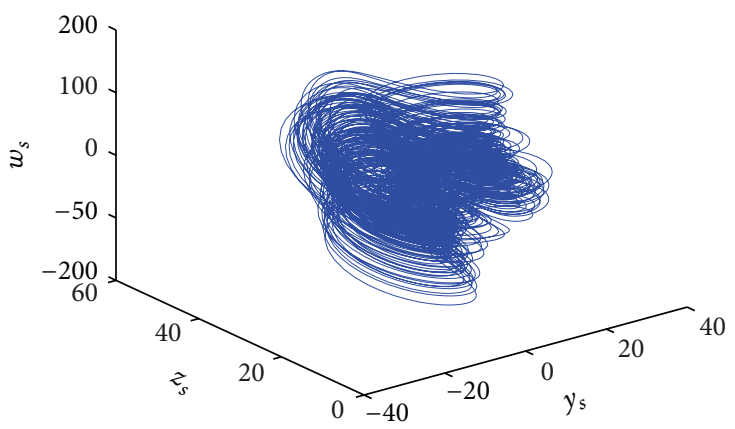

(b) $y_{s}-z_{s}-w_{s}$

FIGURE 7: Phase portraits of the integer-order Lorenz system. 


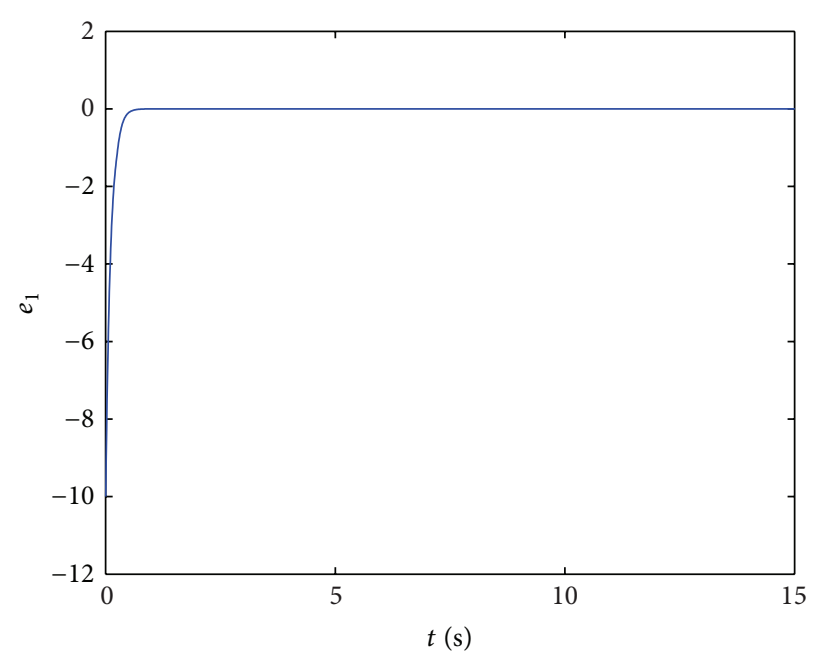

(a) $e_{1}$

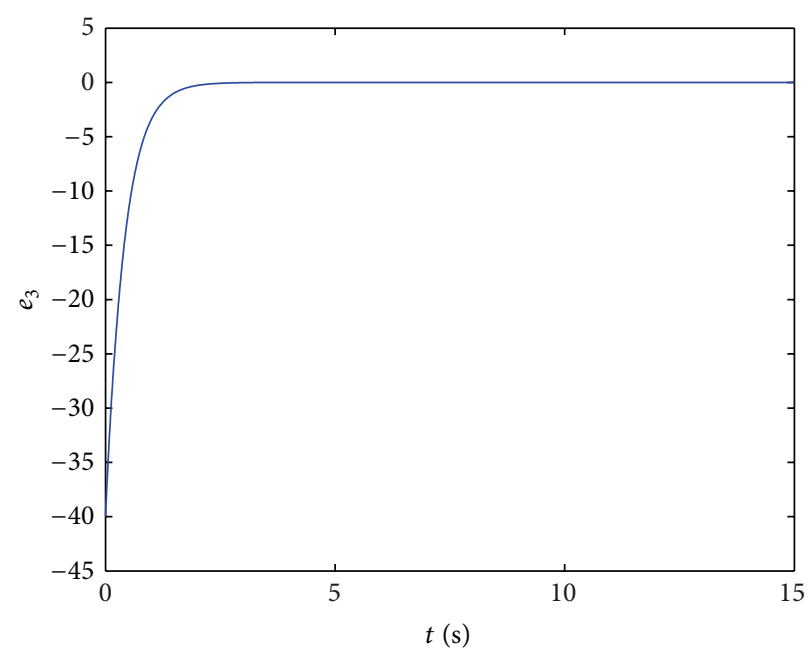

(c) $e_{3}$

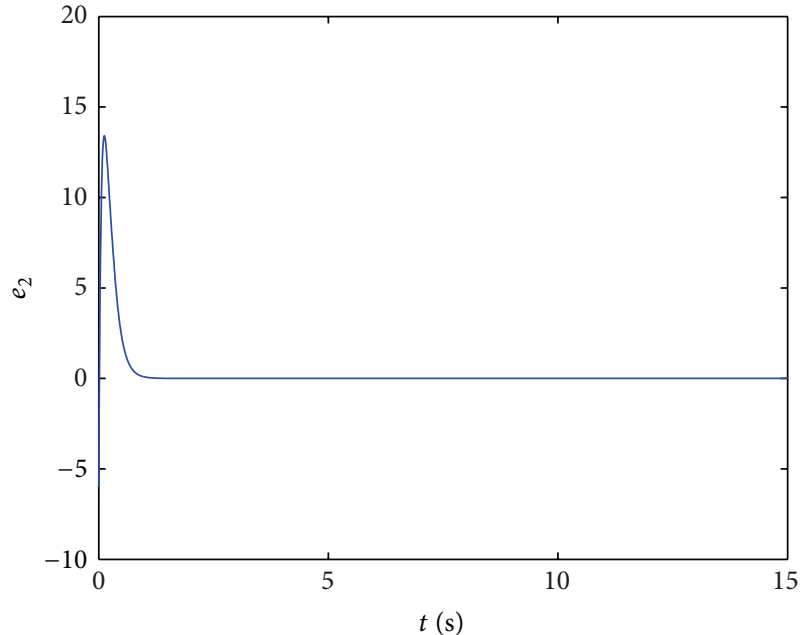

(b) $e_{2}$

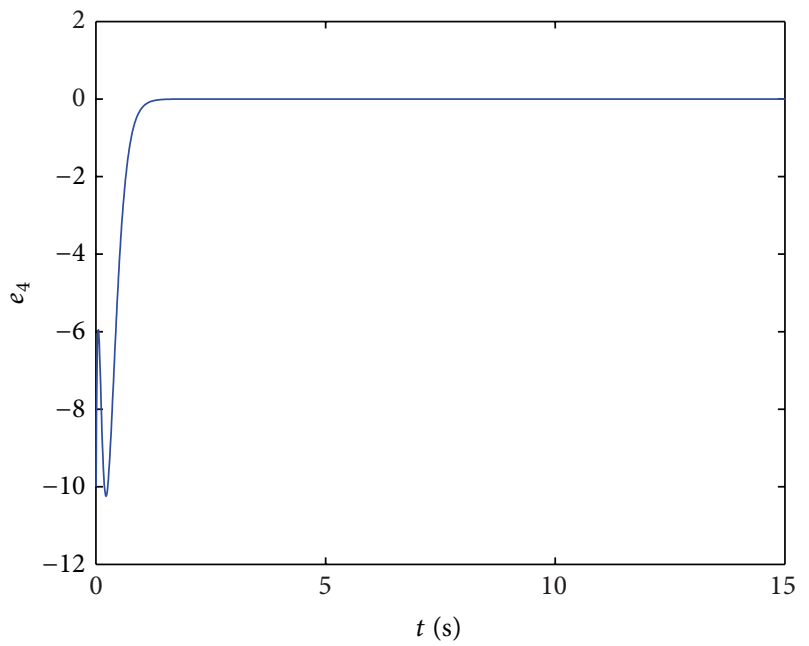

(d) $e_{4}$

FIGURE 8: Synchronization errors between the integer-order Liu system and integer-order Lorenz systems.

and (3) between the integer-order Liu system and integerorder Lorenz system. Numerical results illustrated the effectiveness of the proposed scheme.

\section{Conflict of Interests}

The authors declare that they do not have a direct financial relation that might lead to a conflict of interests for any of the authors.

\section{Acknowledgments}

This wok was supported by the scientific research foundation of National Natural Science Foundation (51109180, 51202200), the National Science and Technology Supporting Plan from the Ministry of Science and Technology of China (2011BAD29B08), the "111" Project from the Ministry of Education of China and the State Administration of Foreign Experts Affairs of China (B12007), Fundamental Research
Funds for the Central Universities (Z109021310), and the scientific research foundation of National Natural Science Foundation (51279167).

\section{References}

[1] L. M. Pecora and T. L. Carroll, "Synchronization in chaotic systems," Physical Review Letters, vol. 64, no. 8, pp. 821-824, 1990.

[2] D.-Y. Chen, W.-L. Zhao, X.-Y. Ma, and R.-F. Zhang, "Control and synchronization of chaos in RCL-shunted Josephson junction with noise disturbance using only one controller term," Abstract and Applied Analysis, vol. 2012, Article ID 378457, 14 pages, 2012.

[3] P. Zhou and W. Zhu, "Function projective synchronization for fractional-order chaotic systems," Nonlinear Analysis: Real World Applications, vol. 12, no. 2, pp. 811-816, 2011.

[4] S. C. Chang, "Synchronization and suppression of chaos in an electromagnetic system," Journal of Vibration and Control, vol. 19, no. 3, pp. 471-480, 2013. 
[5] C.-H. Yang, "Symplectic synchronization of Lorenz-Stenflo system with uncertain chaotic parameters via adaptive control," Abstract and Applied Analysis, vol. 2013, Article ID 528325, 14 pages, 2013.

[6] Y. Chai and L.-Q. Chen, "Projective lag synchronization of spatiotemporal chaos via active sliding mode control," Communications in Nonlinear Science and Numerical Simulation, vol. 17, no. 8, pp. 3390-3398, 2012.

[7] H. N. Pishkenari, N. Jalili, S. H. Mahboobi, A. Alasty, and A. Meghdari, "Robust adaptive backstepping control of uncertain Lorenz system," Chaos, vol. 20, no. 2, Article ID 023105, 2010.

[8] M. Rafikov and J. M. Balthazar, "On control and synchronization in chaotic and hyperchaotic systems via linear feedback control," Communications in Nonlinear Science and Numerical Simulation, vol. 13, no. 7, pp. 1246-1255, 2008.

[9] J. Huang, "Adaptive synchronization between different hyperchaotic systems with fully uncertain parameters," Physics Letters A: General, Atomic and Solid State Physics, vol. 372, no. 27-28, pp. 4799-4804, 2008.

[10] C.-C. Yang and C.-J. Ou, "Adaptive terminal sliding mode control subject to input nonlinearity for synchronization of chaotic gyros," Communications in Nonlinear Science and Numerical Simulation, vol. 18, no. 3, pp. 682-691, 2013.

[11] D. Y. Chen, W. L. Zhao, X. Y. Ma, and J. Wang, "Control for a class of four-dimensional chaotic systems with random-varying parameters and noise," Journal of Vibration and Control, vol. 19, no. 7, pp. 1080-1086, 2013.

[12] D. Chen, W. Zhao, J. C. Sprott, and X. Ma, "Application of Takagi-Sugeno fuzzy model to a class of chaotic synchronization and anti-synchronization," Nonlinear Dynamics, vol. 73, no. 3, pp. 1495-1505, 2013.

[13] S. Bhalekar and V. Daftardar-Gejji, "Synchronization of different fractional order chaotic systems using active control," Communications in Nonlinear Science and Numerical Simulation, vol. 15, no. 11, pp. 3536-3546, 2010.

[14] P. Zhou and R. Ding, "Control and synchronization of the fractional-order Lorenz chaotic system via fractional-order derivative," Mathematical Problems in Engineering, vol. 2012, Article ID 214169, 14 pages, 2012.

[15] R. X. Zhang and S. P. Yang, "Synchronization between fractional-order chaotic systems and integer orders chaotic systems (fractional-order chaotic systems)," Chinese Physics B, vol. 20, no. 9, Article ID 090512, 2011.

[16] J. Bai, Y. Yu, S. Wang, and Y. Song, "Modified projective synchronization of uncertain fractional order hyperchaotic systems," Communications in Nonlinear Science and Numerical Simulation, vol. 17, no. 4, pp. 1921-1928, 2012.

[17] D.-Y. Chen, Y.-X. Liu, X.-Y. Ma, and R.-F. Zhang, "Control of a class of fractional-order chaotic systems via sliding mode," Nonlinear Dynamics, vol. 67, no. 1, pp. 893-901, 2012.

[18] D. Matignon, "Stability results for fractional differential equations with applications to control processing," in Proceedings of the IEEE International Conference on Systems, Man, and Cybernetics (SMC '96), vol. 2, pp. 963-968, 1996.

[19] F.-Q. Wang and C.-X. Liu, "Hyperchaos evolved from the Liu chaotic system," Chinese Physics, vol. 15, no. 5, pp. 963-968, 2006.

[20] X. Wu and Y. Lu, "Generalized projective synchronization of the fractional-order Chen hyperchaotic system," Nonlinear Dynamics, vol. 57, no. 1-2, pp. 25-35, 2009.
[21] R. Zhang and S. Yang, "Robust chaos synchronization of fractional-order chaotic systems with unknown parameters and uncertain perturbations," Nonlinear Dynamics, vol. 69, no. 3, pp. 983-992, 2012.

[22] L. Pan, W. Zhou, L. Zhou, and K. Sun, "Chaos synchronization between two different fractional-order hyperchaotic systems," Communications in Nonlinear Science and Numerical Simulation, vol. 16, no. 6, pp. 2628-2640, 2011.

[23] X. Wang and M. Wang, "A hyperchaos generated from Lorenz system," Physica A: Statistical Mechanics and its Applications, vol. 387, no. 14, pp. 3751-3758, 2008. 


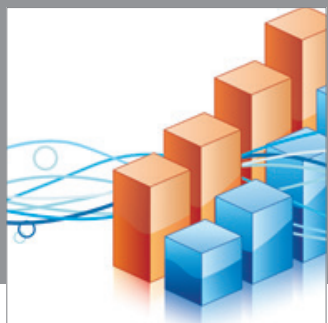

Advances in

Operations Research

mansans

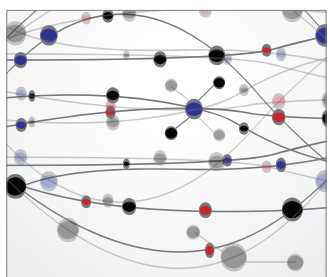

The Scientific World Journal
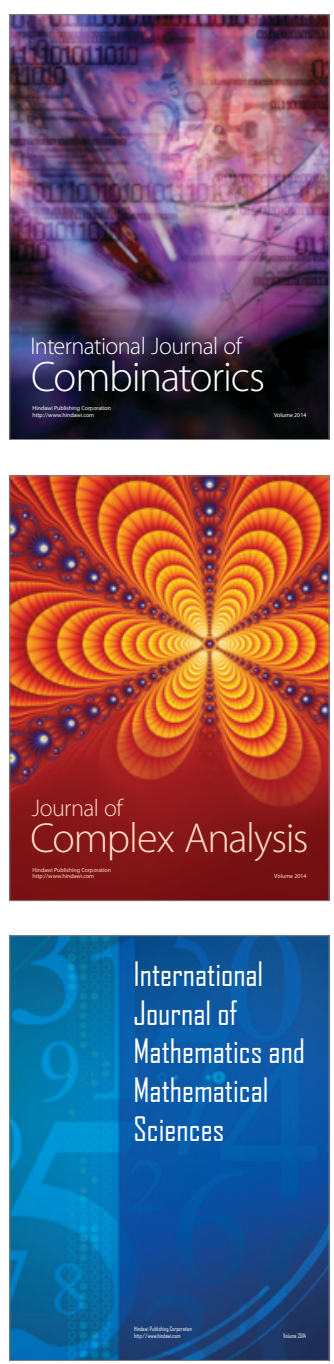
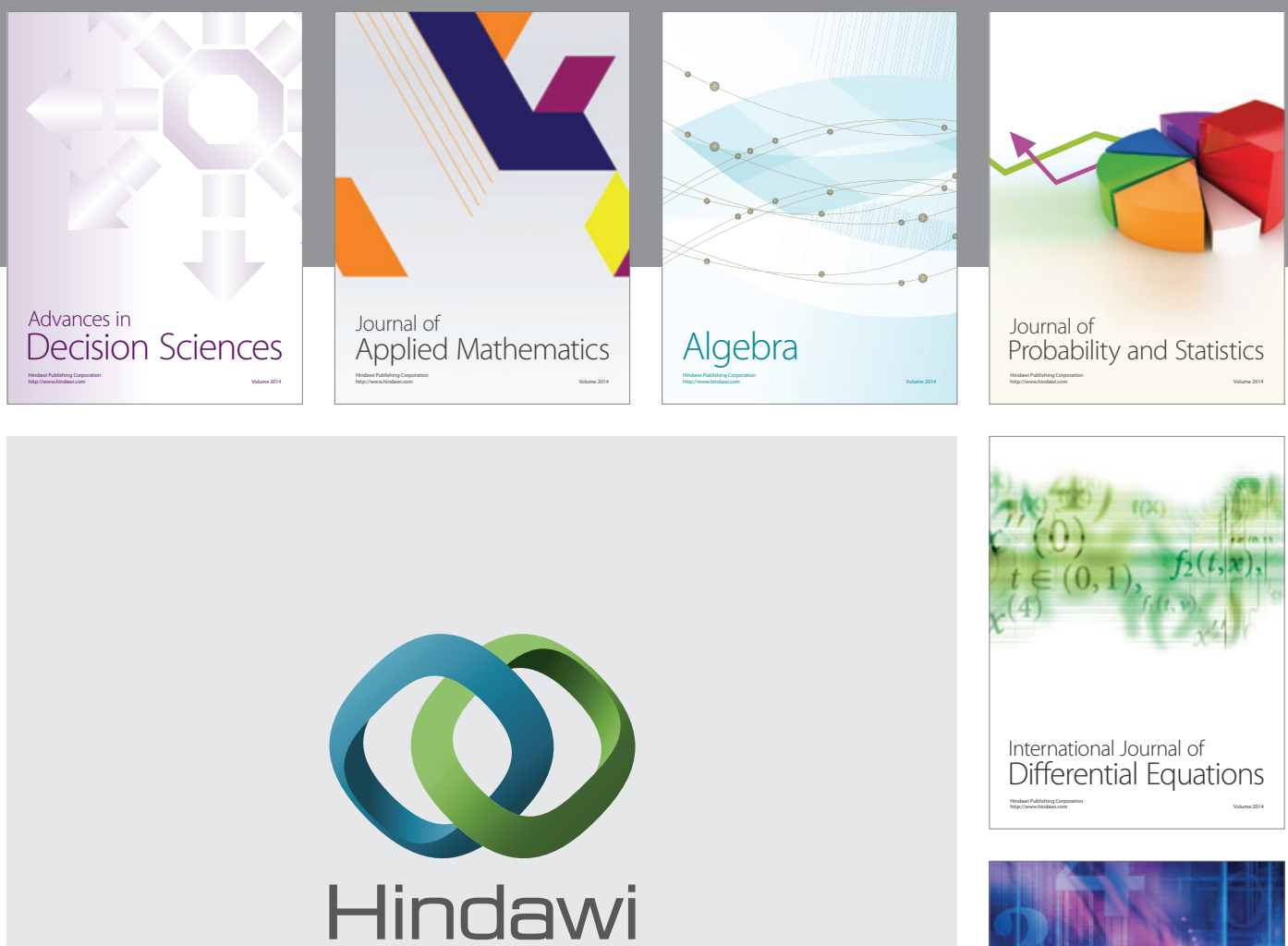

Submit your manuscripts at http://www.hindawi.com
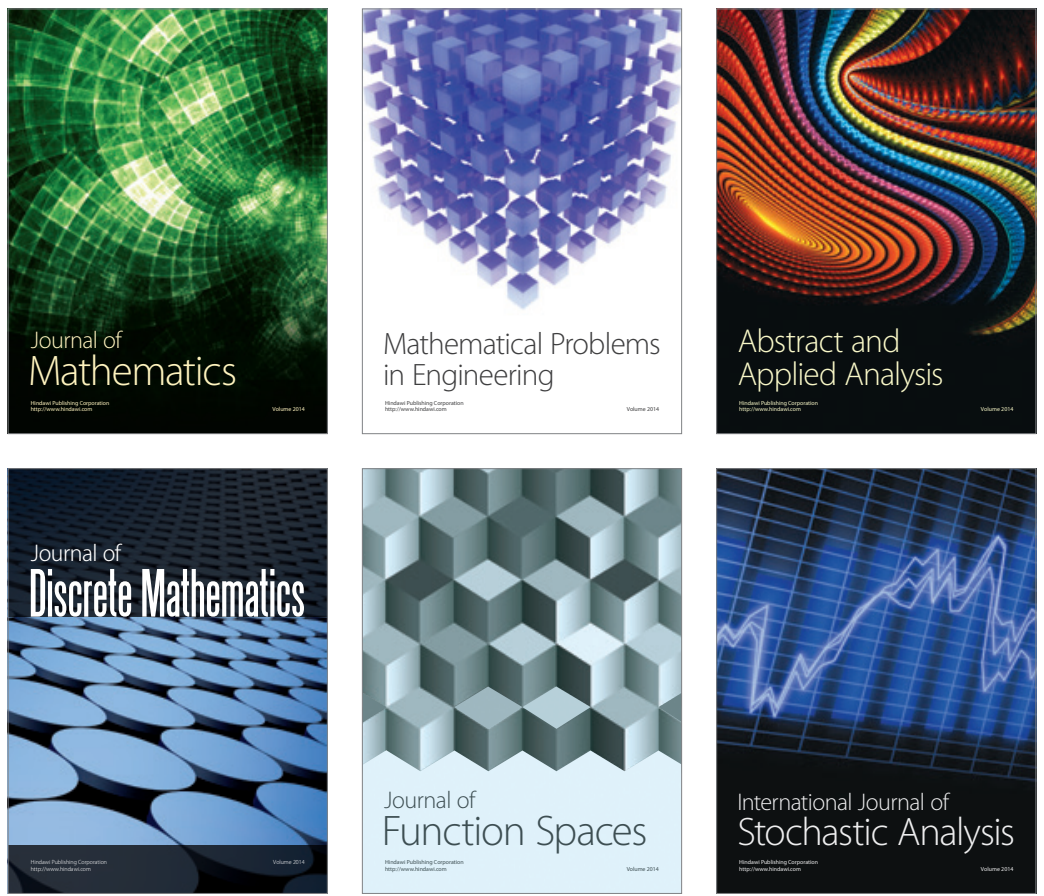

Journal of

Function Spaces

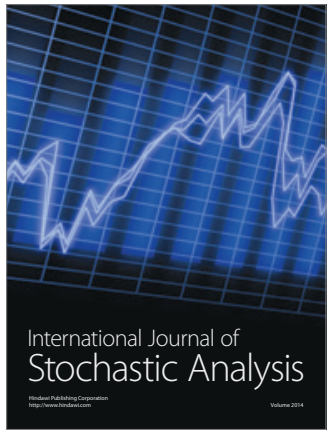

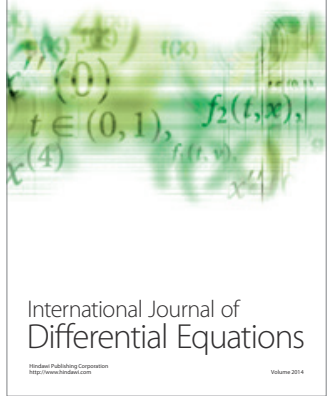
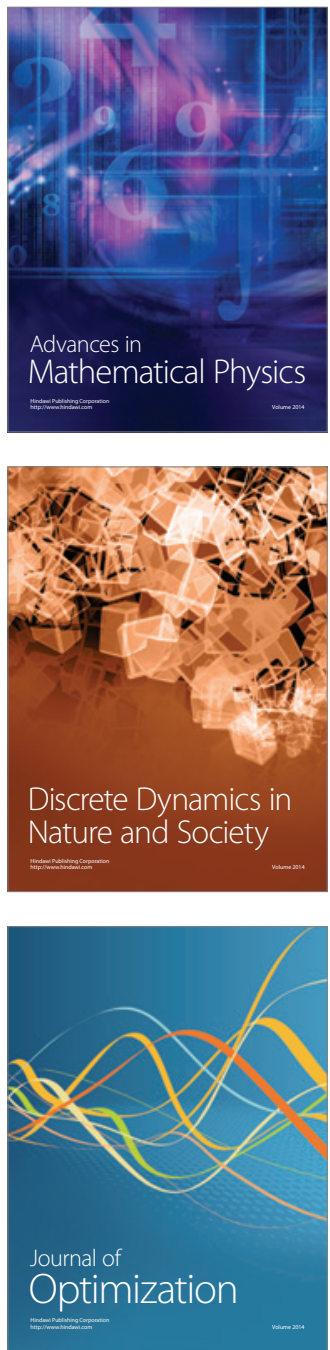